\title{
IR WIRELESS SYSTEM FOR ARCNet LOCAL AREA NETWORK
}

\section{M.J. Betancor}

Univ. de Las Palmas de G.C. Dpt Elec y Telecomunicacion E.T.S.I. Telecomunicacion Campus Universitario Tafira 35017- LAS PALMAS.SPAIN

\section{F.J. Gabiola}

Univ. Politecnica de Madrid Dpt. Ingenieria Electronica E.T.S.I. Telecomunicacion Ciudad Universitaria $\mathrm{S} / \mathrm{N}$ 28040 - MADRID. SPAIN

\section{F.J. Lopez-Hernandez}

Univ. Politecnica de Madrid

Dpt. Tecnologia Fotonica

E.T.S.I. Telecomunicacion

Ciudad Universitaria S/N

28040 - MADRID. SPAIN

\begin{abstract}
In this paper, we show the results obtained with an ARCNet LAN which uses an unguided infrared link as a medium of transmission. This type of systems possesses countless advantages over the conventional coax or $R F$ systems. Besides, when we use IRED, the radiation is negligible for the users and harmless. In the case of possible link tripout, such can be solved by way of software. We studied the effects of shot noise produced by ambient light on the system receiver, and we laid down a parameter, $Z_{b}$, in order to characterize the same. In the same way, we set up a lab prototype of this LAN which gave us satisfactory results. Lastly, we brought together the signals obtained in the laboratory with the layouts and photographs of the sets made. In the sphere of optics, we carried out studies and measurements geared towards filtering the ambient light from the working environment where these local networks were used. Here we show the spectrum characteristics for the film filter used in the course of this kind of work.
\end{abstract}

\section{INTRODUCTION}

The systems which use an unguided medium of communication with infrared present several advantages with respect to the conventional systems [1][2]: they allow for total mobility of the terminal equipment, without loss of direction after change of position, the cost is low, the signal is limited to the volume where it is produced (soundproofing as opposed to similar systems in nearby volumes) and no special training is required for their installation. Thanks to the development in optoelectronic components, we can obtain high bit rate unguided systems at a very low cost.

In the case of links which are to be used inside buildings, the atmospheric attenuation has no effect over the distances covered (1-10 metres).

In the case of a momentary break in the link, the appropriate software can be used to compensate failures at a physical level in the network. This kind of solution is the same as is used in cable systems.

\section{TECHNIQUES USED}

Once the components, the IRED and the photodiodes were chosen, we went on to design the associated electronics: the IRED driver and the pre-amplifier for the receiver.

In the case of the receiver, we carried out extensive study into the noise features of the amplifiers that were to be used. We developed a model which includes the noise produced by the background light with a parameter, $Z_{b}$, similar to the one used to characterize the thermal noise in an amplifier with communication via optical fiber.

On a practical level, we simulated the functions of a local commercial network, ARCNet, on a large layout [3], with optocouplers. Once we had proved that it was possible to work with optical signals, we went on to look at the design, development and layout of a lab prototype which would take advantage of, and improve the system's possible uses as opposed to a coax system.

\section{SENSITIVTTY OF THE RECEIVER}

In the study carried out, we presumed that there could be intersymbol interference $(\gamma)$, a non-zero extinction ratio $(\epsilon)$ of the optical pulse whilst bearing in mind the shot noise produced by the photocurrent, dark current and the ambient or background light current. We defined the parameter $Z_{b}$ to characterise the contribution of the thermal noise of the amplifier and of the dark current and background light shot noise. Its value is:

$$
Z_{b}=\frac{I_{d}+I_{b}}{q} T_{b} \oiint_{0}+Z^{2}
$$


where $I_{b}$ is the current produced by the background light and $Z$ is the typical parameter to characterize the thermal noise in optical communication receivers [4].

The general expression of the pulse $a_{O N}$ which is required in the photodiode and which includes shot noise, thermal energy, and the effect of residual energy when zero value is sent and the fractional energy in the bit interval is:

$$
\begin{gathered}
a_{O N}(1-\epsilon)=Q\left(\frac{h v}{\eta}\right)^{2}\left[\left(\frac{\eta}{h v} a_{O N} \Im_{0}+Z_{b}^{2}\right)^{1 / 2}+\ldots\right. \\
\left.\ldots+\left(\frac{\eta}{h v} a_{O N}\left(1-\gamma^{\prime}\right) \oiint_{0}+Z_{b}^{2}\right)^{1 / 2}\right]
\end{gathered}
$$

where $\gamma^{\prime}=(1-\gamma)$ and the minimum optical power required or sensitivity of the receiver is:

$$
P_{\min }=\frac{a_{O N}+a_{O F F}}{2 T_{b}}-\frac{1+\epsilon}{2 T_{b}} a_{O N}
$$

In figure 1, the minimum optical power is represented by $P_{\min }$ in function of the dark current for various frequencies and resistances. With high resistances (100 $\mathrm{K} \Omega$ ) the thermal noise is negligible and the curves present a marked slope over the range given. In our calculations we saw that with resistances of less than $100 \mathrm{~W}$ the predominant noise is the thermal noise with up to $1 \mathrm{~mA}$ of background current approximately, besides presenting a higher value of $\mathrm{P}_{\min }$. Our calculations were made using $\epsilon=0, \gamma=1$ and $\mathrm{P}_{\mathrm{e}}=10^{-9}$.

\section{SYSTEM IMPLEMENTATION}

The local network chosen was Datapoint's ARCNet for its characteristics: token-passing bus; although this network does not conform to any of the 802 standards within the IEEE or the ECMA it is calculated that there are some 60,000 of these networks in the whole world. What is more, at present Datapoint is striving to add some of the more usual standards [3].

The system which we developed is designed for use in an IBM PC: this limits the feed voltage of the electronic circuits and the consumption within the same. As the logic used in the card for the network ARCNet is TTL [5], this obliges the circuits to follow the same logic. Another inherent feature of the local network is the delay and propagation time from the moment in which the token is sent out until it is received at its

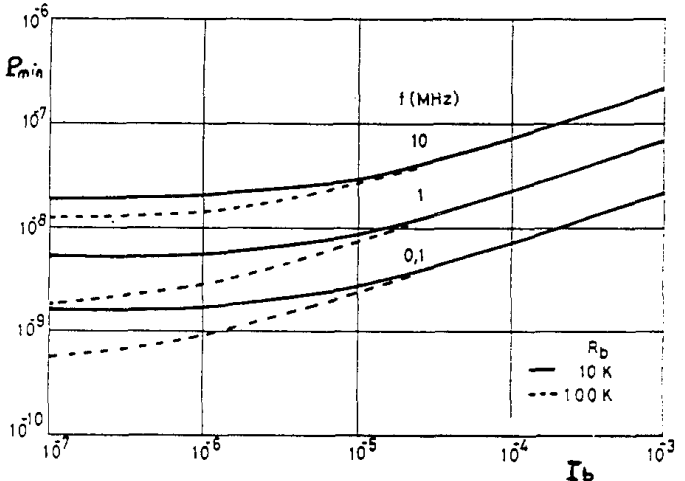

Figure 1.- $P_{\min }$ vs. $I_{b}$ for various frequencies and polarisation resistances. $P_{\min }$ is the minimun power requiered for a given error probability. $I_{b}$ is the current produced for ambient light.

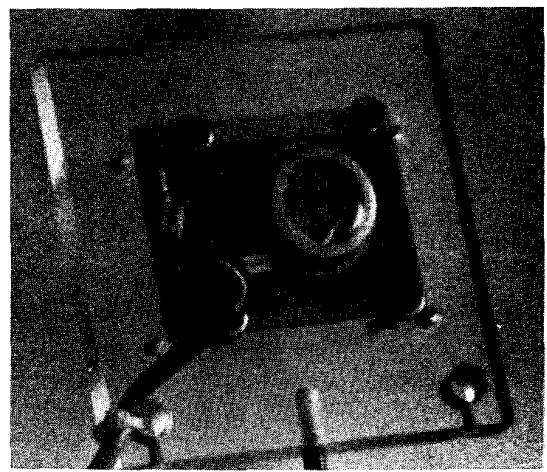

Figure 2.- Lab prototype for the first stage of receiver. PIN-PD and preamplifier. In this case, the receiver does not need additional optics for colecting infrared radiation ( 5 meters).

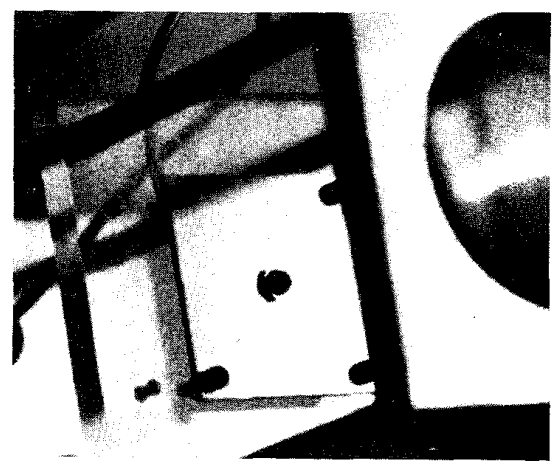

Figure 3.- Prototype of emitter. The IRED (center) is mounted on balanced card. This card enhanced the aligment with the lens. 


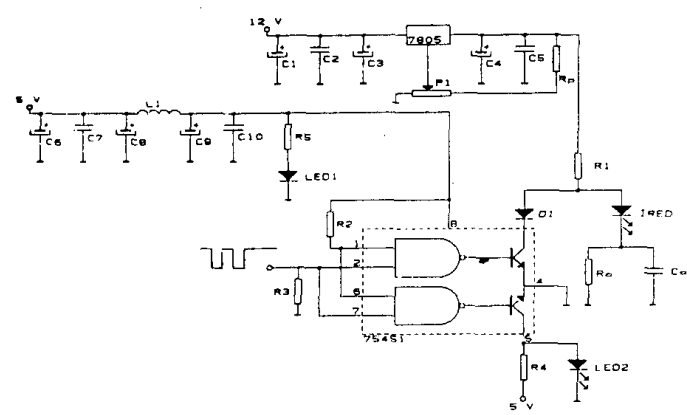

Figure 4.- IRED driver for ARCNet Network. destiny. The binary system of the network is $2.5 \mathrm{Mbit} / \mathrm{s}$ with signals of $25 \%$ of duty cycle: pulses of $100 \mathrm{~ns}$, which signifies a transmission frequency of $10 \mathrm{MHz}$.

In the tests carried out with the local setwork, we did not use optics in the receiver; the distances used (4-5m) and the surface of the PD allow for this kind of set-up.

Figures 2 and 3 are photos of lab prototype of the first stages of the receiver and emitter respectively.

\section{ELECTRONIC CIRCUTTS}

The circuit designed for the transmitter is shown in figure 4.

The receiver uses as its base a transimpedance amplifier on two stages with discrete components. The regenerator uses a comparator which allows for the output of a TTL signal with inputs of $25 \mathrm{mv}$, followed by a monostable side trigger which generates a signal with a duty cycle like the cycle used on the network. The complete circuit of the receiver is to be seen in figure 5 .

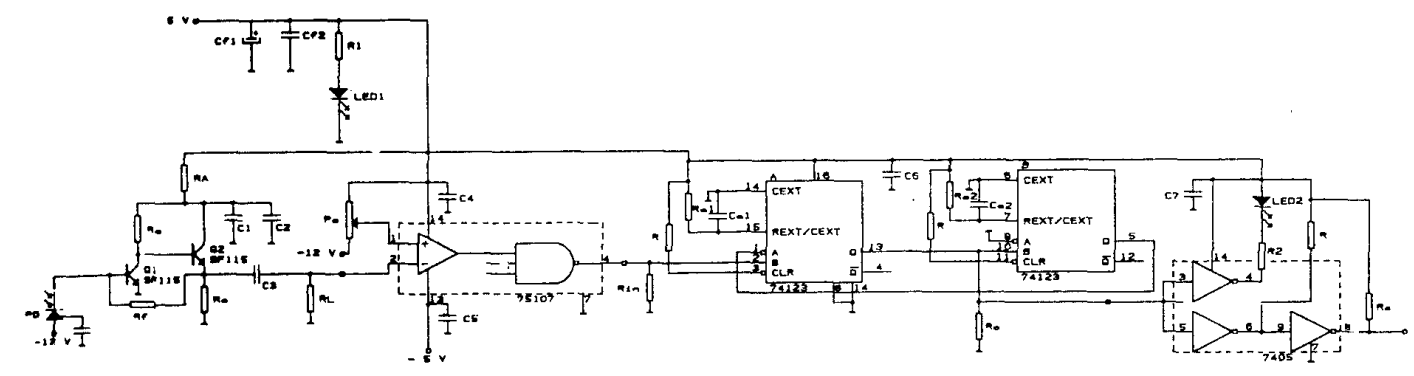

Figure 5. Complete receiver circuit.

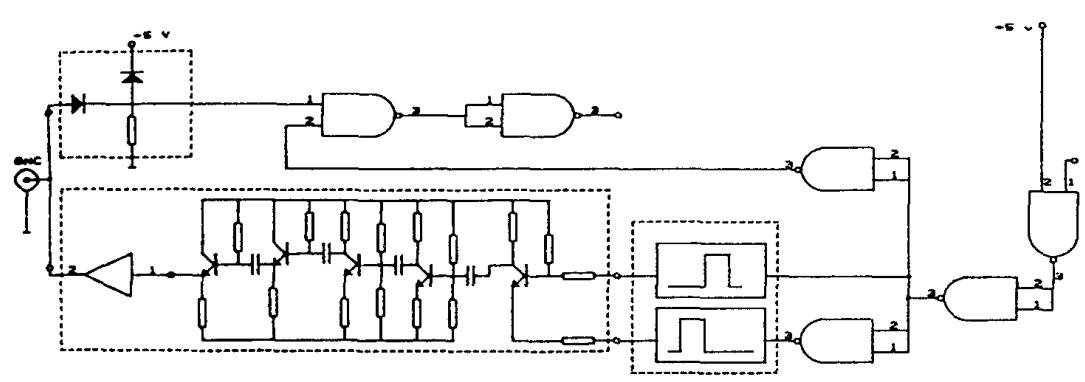

Figure 6.- Cincuit for the conversion of the bipolar signal on the coax line into a unipolar input signal to the optical interface and viceversa. 


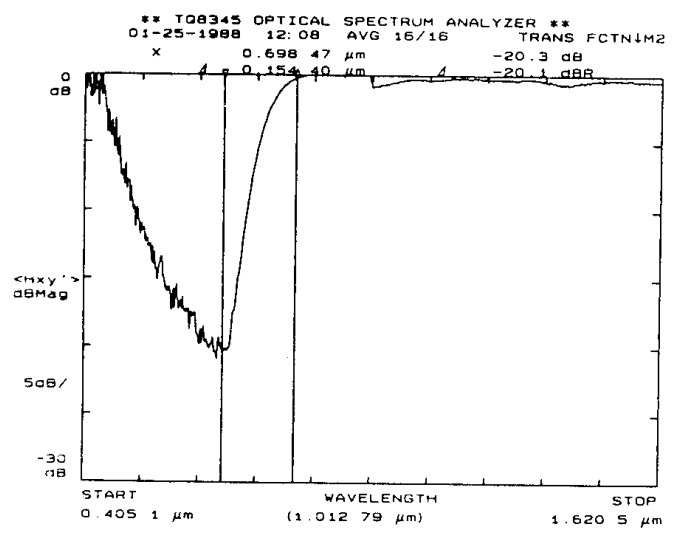

Figure 7.- Spectrum characteristics of a film filter shot.

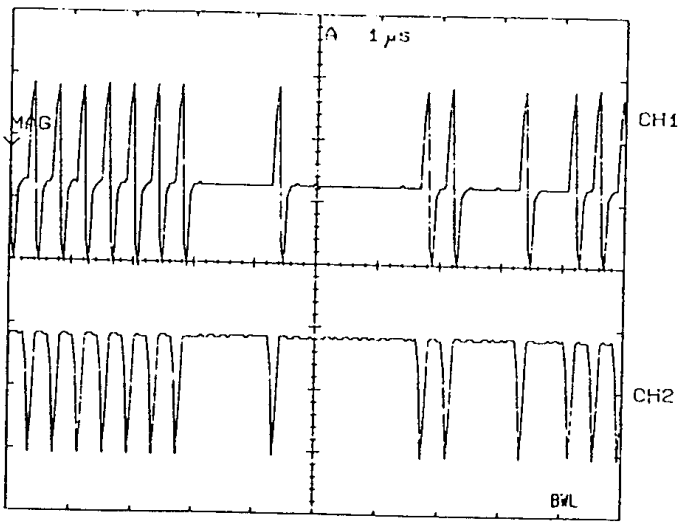

Figure 8.- Wave forms in the coax-optical interface: $\mathrm{CH} 1$ input and $\mathrm{CH} 2$ output.

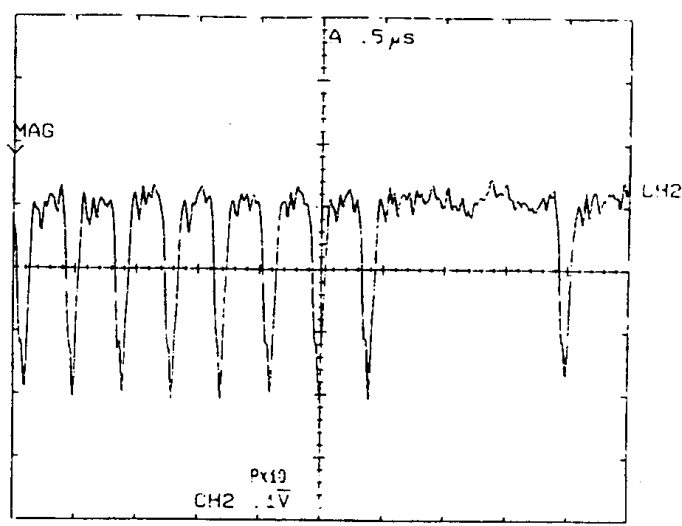

Figure 9.- Signal at the preamplifier output.

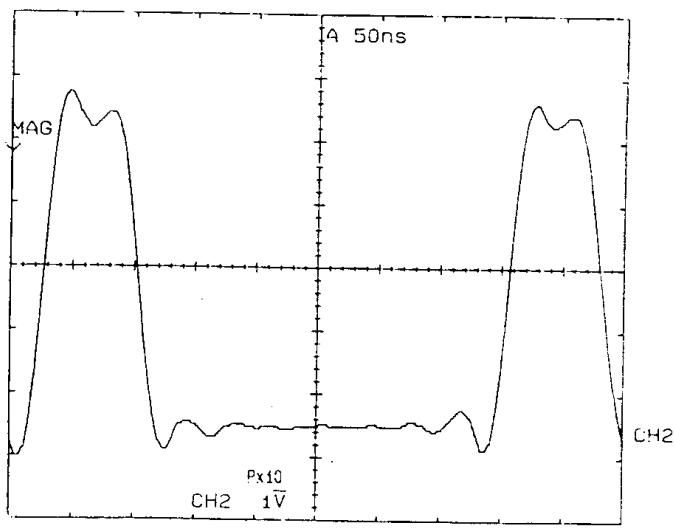

Figure 10.- Final output of the receiver circuit.

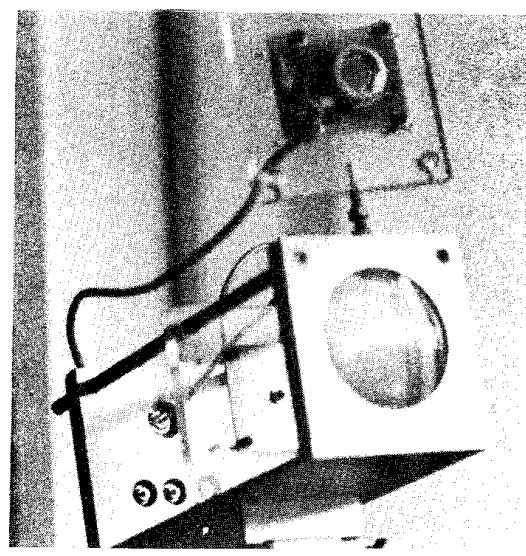

Figure 11.- Lab prototype for optical emitter/receiver. This unit is for each $P C$.

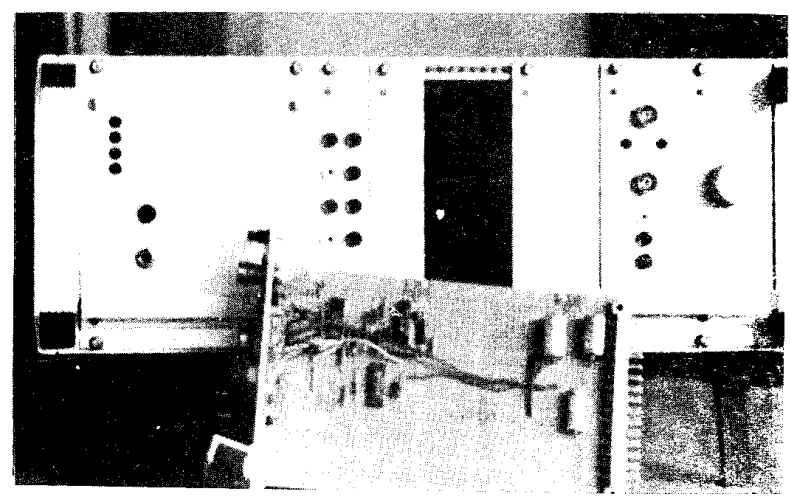

Figure 12.- Electronical interface (IRED driver, amplifier, comparator,...) for ARCNet IR wireless prototype. Bax include IRED current control, power supply ant test points. 
The interfaces between the coax signal and the optical signal were achieved using line interfaces and discrete components, as in figure 6 . Their mission was to adapt the unguided system to cards which were already in existence and which incorporated a hybrid circuit to interface the coax. In future plates, this stage would be integrated for both systems.

To avoid the noise produced by the ambient light [6][7], we used a film filter which was measured in the lab. Its spectrum characteristics are shown in figure 7 . This type of filter is ideal for surroundings where the predominant source of lighting is fluorescent tubes.

In figure $8(\mathrm{CH} 1)$ we show the signal on input of the coax-optical interface and the output of the same $(\mathrm{CH} 2)$. This is the signal which interfaces the IRED driver.

In figure 9, we have the signal to the output of the preamplifier. This form of wave was obtained both with and without ambient and fluorescent light.

In figure 10 , we show the final output of the receiver circuit, which is the monostable and the NOT gates. The signal which is to be passed on to the following stage is almost the same as the original signal in figure 8 .

Figures 11 and 12 are photos of optical emitter/receiver and electronical interface respectively.

\section{CONCLUSIONS}

We show the results obtained with a local ARCNet network which uses an unguided link as a transmission medium. This type of communication can be used for systems of medium high bit rate $(5-20 \mathrm{MHz})$ using the present optoelectronic devices. We should point out that this type of system is low-cost and in certain cases, can work out to be more economical than the conventional system of the coax cable.

The studies carried out as to the characterisation of the shot noise produced by the ambient light in work places and the filtering of this light through simple filters open up the doors for the practical development of these systems. The results obtained at laboratory level back up this claim.
Last but not least, we would like to underline the fact that studies and the necessary designs have been effected to adapt this system both to the cards within the present systems as to the cards of the future.

\section{ACKNOWLEDGMENT}

This work has been supported by Comision Interministerial de Ciencia y Tecnología (CICYT). Project Number: R90 094513.

\section{REFERENCES}

[1] T.E. Bell, "Ethereal Networking Look, Ma, no wires", IEEE Spectrum, Vol: 26 (4), pp 12-12, Apr89.

[2] J.G. Lewis and K. Baker, "Alternative to microwave Infrared wins short-haul run", Data Communications, pp 185-192, Nov-84.

[3] "ARCnet still seeks a standars certificate", Data Communications, Vol: 18 (8), pp 13-13, Jun-89.

[4] S.D. Personick, "Receiver Design for Digital Fiber Optic Communication System (I and II)", Bell Sys. Tech. Jour., Vol: 52 (6), pp 843-886, Jun-73.

[5] Datapoint, ARCNet Designer's Handbook (61610). 1.983.

[6] F.R. Gfeller and U. Bapst, "Wireless In-house Data Communications via Diffuse Infrared Radiation", Proc. of the IEEE, Vol: 67 (11), pp 1474-1486, Nov79.

[7] C.J. Georgopoulos, "Suppresing Background-light Interference in an In-house Infrared Communication System by Optical Filtering", Int. Jour. of Optoelectronics, Vol: 3 (3), pp 247-256, May-88. 